\title{
BMJ Open Transition structures and timing of transfer from paediatric to adult-based care after kidney transplantation in Germany: a qualitative study
}

Jenny Prüfe, ${ }^{1}$ Marie-Luise Dierks, ${ }^{2}$ Dirk Bethe, ${ }^{3}$ Martina Oldhafer, ${ }^{4}$ Silvia Müther, ${ }^{5}$ Julia Thumfart, ${ }^{6}$ Markus Feldkötter, ${ }^{7}$ Anja Büscher, ${ }^{8}$ Katja Sauerstein, ${ }^{9}$ Matthias Hansen, ${ }^{10}$ Martin Pohl, ${ }^{11}$ Jens Drube, ${ }^{1}$ Florian Thiel, ${ }^{12}$ Susanne Rieger, ${ }^{13}$ Ulrike John, ${ }^{14}$ Christina Taylan, ${ }^{15}$ Katalin Dittrich, ${ }^{16}$ Sabine Hollenbach, ${ }^{17}$ Günter Klaus, ${ }^{18}$ Henry Fehrenbach, ${ }^{19}$ Birgitta Kranz, ${ }^{20}$ Carmen Montoya, ${ }^{21}$ Bärbel Lange-Sperandio, ${ }^{22}$ Bettina Ruckenbrod, ${ }^{23}$ Heiko Billing, ${ }^{24}$ Hagen Staude, ${ }^{25}$ Reinhard Brunkhorst, ${ }^{26}$ Krisztina Rusai, ${ }^{27}$ Lars Pape, ${ }^{1}$ Martin Kreuzer ${ }^{1}$

To cite: Prüfe J, Dierks M-L, Bethe $\mathrm{D}$, et al. Transition structures and timing of transfer from paediatric to adult-based care after kidney transplantation in Germany: a qualitative study. BMJ Open 2017;7:e015593. doi:10.1136/ bmjopen-2016-015593

- Prepublication history and additional material for this paper are available online. To view these files please visit the journal online (http://dx.doi. org/10.1136/bmjopen-2016015593).

Received 22 December 2016 Revised 24 March 2017 Accepted 27 March 2017

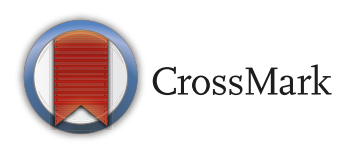

For numbered affiliations see end of article.

Correspondence to

Dr Jenny Prüfe; pruefe.jenny@ mh-hannover.de

\section{ABSTRACT}

Objectives It is known that transition, as a shift of care, marks a vulnerable phase in the adolescents' lives with an increased risk for non-adherence and allograft failure. Still, the transition process of adolescents and young adults living with a kidney transplant in Germany is not well defined. The present research aims to assess transitionrelevant structures for this group of young people. Special attention is paid to the timing of the process.

Setting In an observational study, we visited 21 departments of paediatric nephrology in Germany. Participants were doctors $(n=19)$, nurses $(n=14)$ and psychosocial staff $(n=16)$ who were responsible for transition in the relevant centres. Structural elements were surveyed using a short questionnaire. The experiential viewpoint was collected by interviews which were transcribedverbatim before thematic analysis was performed.

Results This study highlights that professionals working within paediatric nephrology in Germany are well aware of the importance of successful transition. Key elements of transitional care are well understood and mutually agreed on. Nonetheless, implementation within daily routine seems challenging, and the absence of written, structured procedures may hamper successful transition.

Conclusions While professionals aim for an individual timing of transfer based on medical, social, emotional and structural aspects, rigid regulations on transfer age as given by the relevant health authorities add on to the challenge.

Trial registration number ISRCTN Registry no 22988897; results (phase I) and pre-results (phase II).

\section{INTRODUCTION}

Transition in medical care is a high-risk period in adolescence and young adulthood. ${ }^{1}$ In 2014, a report from the Institute of Medicine prioritised transition from paediatric to adult healthcare as a key issue to improve the

\section{Strengths and limitations of this study}

- This study is the first nationwide report to explore transition structures in Germany including all departments of paediatric nephrology in the country which offer dialysis and transplantation in the paediatric population

- Although working with subjective impressions, we strengthened the credibility of this study by interviewing individuals with different professional backgrounds (medical doctors, nurses, psychosocial staff) to gain multiple perspectives on the system researched.

- Furthermore, we compared and contrasted participants' personal perceptions with data from a survey which was conducted in the very same departments.

- Still, as exploratory qualitative research, the findings are limited to the population researched (professionals working in paediatric nephrology in Germany), and we cannot extrapolate our results to other geographical regions. Further research is needed to judge on the transferability of the results into other settings.

- Another limitation is that due to the design of the study, we could only focus on the perspectives of professionals working in paediatric nephrology. Insights from adult nephrologists as well as from the patients' themselves are lacking but will be explored by our working group in due course.

health of young adults with chronic diseases. ${ }^{2}$ Annually, about 60 young people living with a kidney transplant (KTx) have to transfer from paediatric into adult care in Germany and Austria. ${ }^{3}$ The transition process can be considered successful if the adolescents concerned are enabled to be as self-competent and 
independent as possible in all relevant matters of their disease and care, while achieving satisfactory quality of life. $^{4}$

Yet, transfer also is a discontinuation of care and marks a vulnerable phase in the adolescents' lives with an increased risk for non-adherence and allograft failure. ${ }^{5}$ Some studies report unexpected loss rates in kidney grafts to be as high as $24 \%-42 \%$ within 3 years after transfer. ${ }^{6-8}$ Transplant failure, however, forces the young patient back to dialysis which in turn reduces quality of life and increases morbidity and mortality as well as healthcare expenses. $^{5}$

To date, the transition process of adolescents and young adults with KTx is not well defined. Therefore, this observational study aims to assess the transition-relevant structures from the providers' perspective, taking into account both a statistical as well as an experiential viewpoint. Special attention is paid to the question of timing the transfer and reasons to delay it.

\section{METHODS}

As part of the TRANSNephro study, we evaluated the existing structures relevant to transitioning young people with a transplanted kidney living in Germany and Austria from a paediatric perspective. The study has been registered at ISRCTN Registry (no 22988897), and ethics approval was obtained from the relevant committees at all study sites. The study protocol was published on December 2014. ${ }^{9}$

Our research team visited all 21 centres of paediatric nephrology where children and adolescents after KTx are treated in Germany and one in Austria. Those centres cover more than $99 \%$ of paediatric care in this field. A questionnaire on patients and staff characteristics as well as on aspects of transition was answered by a member of the medical team in each centre. ${ }^{3}$ Furthermore, we conducted face-to-face in-depth interviews with paediatric nephrologists, nursing staff and members of the psychosocial teams across all centres. Each centre decided on its own which members of the team were most competent to report on transition. Only those professional groups that were involved in transition in the relevant centres were represented by an interviewee. In three centres, medical doctors could not participate due to time constraints.

The interview guidelines were developed based on findings from a systematic literature review ${ }^{10-13}$ as well as from team discussions within our own department. Each interview consisted of four parts:

1. the description of the actual procedure as perceived by the relevant staff,

2. the identification of exemptions and distinctive features,

3. reflections on strengths and difficulties of the work protocol, and

4. future outlook on possible changes and recommendations on transition.
Participants were informed that the interviews aimed to collect experiential viewpoints on transition after paediatric kidney transplantation from various staff perspectives.

All interviews were conducted, digitally audio recorded and transcribed verbatim by the same psychologist (JP). Additionally, contextual details were protocolled after each interview. Transcripts were analysed using thematic analysis. Coding was done independently by JP and MK and then discussed in the working group and representatives of the participating centres to enhance the analytical process and derive the themes. Coding examples from the interviews are available in online supplementary data.

Anonymised information regarding each patient's medical care and treatment course was registered for all patients who were transferred out from the participating paediatric nephrology centres in the years 2011 and 2012, covering the last 12 months before transfer. Details on the exact protocol and data were previously published by our working group and include additional data from Austria. ${ }^{3}$ Austrian data were not included in this present analysis because of differences in the healthcare systems between Germany and Austria.

The questionnaire and interview guidelines as well as the coding tree are available on request. See supplementary material for COREQ checklist.

\section{RESULTS}

\section{Existing transition structures}

In addition to the 21 questionnaires that we collected from all participating departments, 49 interviews (paediatric nephrologists 19, nurses 14 and members of the psychosocial teams 16) were compiled for the analysis. On average, interviews lasted $22 \mathrm{~min}(10-80 \mathrm{~min})$. Interview data of adult nephrologists will be analysed and published separately because of the huge amount of data obtained.

Most of the participating centres belong to the non-profit organisation Kuratorium für Dialyse und Nierentransplantation (KfH) (15/21). All of those are associated with university hospitals $(11 / 15)$ or large community hospitals (4/15). Five are units of independent university hospitals, and the remaining one is a large regional hospital. The median number of patients in long-term care after kidney transplantation was 30 per centre (range 3-120). Within the past years, the number of transferred patients averaged four per centre and year (range 0-10) and amounted to a total of 111 patients across Germany. Detailed characteristics of this cohort have been published before. ${ }^{3}$

In the questionnaire, the reporting staff of 16/21 (76\%) centres claimed to apply a transition procedure which was mutually agreed on even if it was unwritten. However, the interviews with different members of staff revealed that what was thought to be an internal agreement was interpreted differently by team members. Furthermore, procedures were not consequently applied in each patient, leaving doubts regarding the claimed unwritten agreements. Only three of $21(14 \%)$ centres reported the 
use of checklists and standard operating procedures to structure the transition process.

The commonly stated aim of the transition process was to support children's development with regard to selfcare and autonomy. To achieve this, the main elements reported by all centres were as follows:

- to call in the patient for consultation on their own and without their parents

- to ask the patient about his/her drug schedule

- to ask the patient about his/her blood results

- to ask the patient about his/her blood pressure and weight monitoring

- to ask the patient to search for a nephrologist when transfer is approaching.

To further assist the transition process, educational offers were made. Of 21 centres, 17 (81\%) encouraged their patients to participate in a specialised training programme for children with chronic kidney disease that is provided by the KfH ('Endlich Erwachsen'/finally grown-up). ${ }^{14}$ Median number of participants was two per year-independently of the centre's total number of patients with KTx. Transition-specific in-house courses were offered in 2/21 (10\%) centres only. Nineteen of $21(90 \%)$ centres offered in-house training courses on selected topics or patient camps, yet those not being transition specific. None of the centres used tools to assess transition readiness of the patients. Neither did any centre apply specific transition tools, like patient or family questionnaires, computer programs or web-based patient training and information systems.

In the survey, doctors stated that the transition process as the period of time used to deliberately prepare young patients for transfer was initiated at age 14-16 in $9(42 \%)$ centres; another 9 (42\%) commenced at age 16-18. Only three $(14 \%)$ centres reported to starting the transition process in children younger than 14 . Yet, a comparison of interview data with the questionnaires revealed incoherence in 8/21 centres: data differed by 2 years, and in practice, start of transition was at higher age than aimed at. One centre stated to start the process of transition at the early age of 12 years 'if we remember'.

Staff covered different roles and functions within the transition process. Nephrologists felt responsible for the medical work-up and organisation of the transfer, as well as for training the patient in relevant matters such as medication and health literacy. Nurses coordinated appointments and supported patients in their disease management. Tasks of the psychosocial staff included help with schooling and education, aspects of general social work and crisis intervention.

When the time of transfer approached, patients and their families were asked to search for an adult nephrologist and if necessary a transplant centre. In all centres, the physicians made sure that blood results were up to date and the annual follow-up was completed. They then prepared a letter for the adult nephrologist with the patient history and relevant details. Only $5 / 21$ physicians
(24\%) reported that they generally aimed for a telephone contact with the future nephrologist before transferring out the patient. One paediatrician and two psychologists from three different centres regularly accompanied their patients for their first appointment in adult clinicsusually when transferred internally or within town. Eight of 21 (38\%) departments had experience with 'shared care', three of which only occasionally offered it in individual cases of local or in-house transfer. The period of shared care varied from effectively having only one follow-up appointment after the first contact to the adult nephrologist was made (two centres) up to 1 year of alternating appointments (two centres).

In all centres, an informal offer to contact the paediatric unit in case of occurring questions was made to the patient. A standardised medical follow-up history was not carried out in any of the centres; neither was a scheduled consultation with the adult nephrologist. However, $3 / 21$ physicians $(14 \%)$ said that they were interested in a follow-up and generally asked the adult nephrologists to report back to them; yet, this was rarely done.

Transfer clinics with both adult and paediatric nephrologist attending did not take place, and the involvement of specialist liaison nurses to ensure continuity of care was wished for yet not established in any department. All participants stated that due to shortage of staff, financial constraints, administrative barriers and lack of time these aspects could not be realised.

\section{Timing the transfer}

The age at transfer was subject to regulation in 16/21 centres $(76 \%)$ and defined as age 18 years. In another four departments, there was an administrative recommendation to transfer at age 18 which was not binding. Only one centre stated not to have any such regulations. However, as most centres applied for individual exemptions to extend paediatric care beyond age 18, the average age at transfer in the retrospective cohort was slightly higher (18.3 years; range $16.5-36.7$ ). The oldest patients to be transferred out suffered multiorgan disease and complex disability. The relevant centre reported that they were unable to find adult departments able to care for patients with such complex conditions. Half of the centres applied for exemptions on a regular base. Extension of paediatric care was granted mainly for 3-6 months. Four of 21 (19\%) centres reported to frequently apply successfully for 3 years of extension or until education is completed. One centre stated that if extension is granted, this was done up to the age of 25 . Reasons to apply for exemptions were both of medical and psychosocial nature. Whether exemptions were granted varied greatly depending on regional healthcare policies.

In the interviews, every participant challenged the expected transfer age of 18 years. There was mutual agreement that such regulation was counterproductive and impeded an individualised care and a transfer was scheduled according to patients' skills and needs. 
As a prerequisite for transfer, doctors, nurses and psychosocial staff wanted patients to be (a) securely enrolled in professional training, (b) knowledgeable about their disease, (c) competent in self-care, (d) emotionally resilient and (e) with stable allograft function. As a worst-case scenario, participants commonly described a situation when a patient was undergoing several changes at the same time, particularly if this involved changing both the local paediatrician as well as the nephrologist simultaneously:

In a worst case, we are forced to transfer a patient at his 18th birthday. At the same time his local paediatrician will transfer him as well. [After completion] of school he will then have a job offer in another place and will need to change doctors again. After completion of his training, he will find a job at yet another place... so, technically it will be all about transfer for years. Yet, the patient needs some continuity, caring hands which hold him tight while everything keeps changing, and a place where he feels secure. This is why we apply for extended paediatric care until everything is settled

Looking closer at arguments to extend paediatric renal care, reasons could be grouped into four categories: (1) medical reasons, (2) social factors, (3) patient personality and skills and (4) structural aspects.

\section{Medical reasons}

The first and foremost medical reason was allograft function. All participants stated that transfer is postponed if there is creeping creatinine, new detection of donor-specific antibodies or acute rejection episodes. All centres aimed for a stable kidney function within that last 6-12 months prior to transfer. In practice, serum creatinine levels increased statistically significant over the final year in paediatric care, as previously published by our workgroup. ${ }^{3}$ In addition, $22 / 111$ patients $(20 \%)$ were actually transferred with an increase of serum creatinine $\geq 20 \%$ as compared with baseline at the final paediatric visit in 2011 and 2012. Centres reported that pressure from hospital administration as well as from the local health authorities forced them to act against their medical conviction.

Approaching dialysis in contrast did not conflict with transfer; rather, it was regarded as a clear cut and a newly starting treatment with a new team.

Some participants claimed that Orphan diseases or complex syndromic conditions were a reason to postpone transfer, particularly if there was no adult nephrologist available who felt competent and confident in dealing with such disease.

It was further complained that structures to support patients with complex syndromic conditions after transfer were lacking and that it was "a hopeless situation which is causing headaches when you don't know where to transfer to because there is no such structure for the multiple disabled patient. You simply don't know where to transfer to."
Learning disability and developmental delay as reasons to postpone transfer were viewed controversially. In some departments, a significant developmental delay was regarded as a good reason for continuing paediatric care given that, by developmental status, these patients' needs were more child than adult like. Also, severe developmental delay was the argument most commonly accepted by health authorities to postpone transfer. Furthermore, it was stated that, based on personal experience, adult nephrologists would dislike accepting such patients for treatment and prefer them to stay in paediatrics as well. Other departments, however, would transfer such patients subject to their social environment arguing that prolonging paediatric care would not change patient's competence or mental abilities. Again, it was seen critical if multiple disabled young people had to transfer out from different clinics such as nephrology+neurology+metabolics at the same time changing the complete set of medical teams at one point in time, namely, the patients' 18 th birthday.

\section{Social factors}

Social reasons included aspects of family support, social network, as well as school, training and work environment. It was commonly agreed on that by the time of transfer, schooling should be completed and job training well settled at least. It was argued that the support provided by social workers in the paediatric departments was not available in adult units yet desperately needed with regard to choosing and finding a job or training placement. Furthermore, it was pointed out that many social problems start after leaving school. Teachers and pupils were described as considerate, knowing the young person and the effects of the disease on the patients' everyday life. In contrast, it was commented that when entering the labour market, pressure tended to rise and the work environment was regarded as less accommodating to the relevant needs, leading to social exclusion of the chronically ill adolescent.

And again, there was concern about too many changes at the same time since: "Job training brings in so many changes and new information. It's like flooding on the adolescent patient and they have to master this all. Then adding a transfer, this would be simply too much. I mean, even healthy people fail with this."

A key question appeared to be as follows: How much support does a patient need in dealing with everyday hassles and is the social environment sufficiently supportive? Particularly, when critical life events accumulated, for example, leaving school, moving out of home, parental divorce and others, this was considered bad timing for transfer.

\section{Individual aspects}

On an individual base, aspects to be considered regarding readiness for transfer were autonomy and self-responsibility, emotional stability and cognitive maturity, disease-related knowledge and adherence. The 
underlying thought was that patients need to be able to manage the disease and disease-related needs in order to maintain health status and allograft function after transfer.

Participants stressed the role of emotional stability as a prerequisite to act self-responsibly and maturely. This included accepting the chronic disease as part of one's personal history and being. However, it was also perceived that the emotional development of the chronically ill patient was somewhat delayed, especially when patients were well mothered and protected by parents. Yet, these statements were solely based on personal impressions of medical staff and psychologists. No centre confirmed the use of standardised assessment to evaluate emotional stability.

A lack of emotional stability was regarded a risk factor for non-adherence and consequently a poorer outcome which is why postponing transfer in such cases was seen as vital. Still, one nurse commented that, unfortunately, it frequently appeared to be those patients least self-responsible and most at risk who urged for timely transfer. It was assumed that this type of patients felt overprotected and under control and wished to escape the close supervision of paediatricians and nurses through entering adult care.

Table 1 IPNA consensus statement and its realisation in Germany

\begin{tabular}{lll}
\hline 1. Transition to transfer & Aimed for by & Fully applied by \\
\cline { 1 - 1 } Delivery of necessary patient care information to the receiving adult service & $21 / 21$ & $21 / 21$
\end{tabular}

2. Transfer from paediatric to adult nephrology should...

be individualised for each patient after s/he has completed a transition plan $21 / 21$

$1 / 21$

depending on completion of physical growth and educational, social and

psychological attainment

be agreed on jointly by the patient and his/her family/carers in conjunction $\quad 21 / 21$

with the paediatric and adult renal care teams

take place during a period without crises, especially if there is unstable social 21/21 $21 / 21$ support

take place after completing school education

$21 / 21$

$17 / 21$

take into account treatment plans by other subspecialties, with particular reference to urological supervision

take place with due consideration of financial factors and not be done $\quad 21 / 21 \quad 21 / 21$ abruptly without adequate preparation as a result of financial pressures

introduction to the concept of transition in early adolescence (12-14 years)

information about transition in a gradual manner appropriate to his/her

$21 / 21$

developmental stage and intellectual ability

$21 / 21$

identified lead clinicians (transition champions) in paediatric and adult units $\quad 21 / 21 \quad 0 / 21$

to coordinate and educate on transition issues

a nominated key worker responsible for coordinating transition from both the $21 / 21 \quad 0 / 21$

paediatric and adult renal service

a generic transition plan that then can be individualised for each patient $\quad$ 21/21 $\quad 1 / 21$

involvement of parents, other family members and even boyfriends/girlfriends 21/21 $21 / 21$

the opportunity of an informal visit to the nominated adult service before $\quad 21 / 21 \quad 21 / 21$

transfer occurs

the opportunity to participate in group sessions with other young people who $21 / 21$

are about to transition for peer-support experience

$21 / 21$

3. Transition or transfer clinic

$\begin{array}{lll}\text { with both adult and paediatric nephrologist in attendance } & 21 / 21 & 0 / 21\end{array}$

with specialist nurses for adult patients who liaise with specialist nurses from no data $\quad 0 / 21$

the paediatric unit can ensure continuity of care

providing a comprehensive written and verbal summary of all the $\quad 21 / 21 \quad 0 / 21$

multidisciplinary aspects of the young person's care including medical,

(summary available but nursing, dietary, social and educational information

not as part of transition clinic)

offering a transition pathway to assert their autonomy and help provide the $\quad 21 / 21 \quad 0 / 21$ relevant information about themselves

Not standardised

IPNA, International Pediatric Nephrology Association. 
In addition to those patient-related factors, several professionals pointed out that it was also the parents who needed to learn to let go and leave paediatrics behind. There was mutual agreement that, ideally, a consensus should be reached with patient and parents about the readiness to transfer.

\section{Structural aspects}

Although psychosocial reasons were considered essential aspects to account for in the transition process, it varied greatly whether these factors were regarded as valid arguments by the health authorities who had to decide on postponing transfer. Medical reasons in contrast were generally accepted.

Simultaneously, it was pointed out that there was a lack of professional psychosocial support for emotionally challenged patients outside paediatric nephrology. Across Germany, participants stated that adult nephrology clinics would not provide psychologists or psychotherapists. In rare cases, social workers were said to be available to help with legal aspects. This was confirmed in a survey among adult nephrologists (unpublished data) as well as by the $\mathrm{KfH}$ administration. Yet, waiting time for locally established psychotherapists was experienced to mount up to 6-9 months for regular therapeutic appointments; this was regarded unreasonable in the face of the patients' histories.

It was also reported that sometimes transfer took place irrespective of the patient's situation but due to structural requirements. Reasons for this were lacking staff and equipment in relation to the total number of patients as well as funding cuts. Participants across disciplines stressed that, in times of financial restrictions, psychosocial support and speaking medicine were the first to be cut despite their utmost importance for the patients' rehabilitation. In some cases, fatal consequences were reported: "Cause transferring patients who are not yet ready will lead to premature organ loss! I remember some patients who we thought needed more support and training. But extension was not granted. That didn't go well at all! One of them went to heaven."

To summarise, we compared our findings with the recommendations of the International Pediatric Nephrology Association (IPNA) consensus statement on transition and transfer. ${ }^{15}$ Table 1 shows if and to what extent the suggested criteria were met by the participating departments of the present research.

\section{DISCUSSION}

TRANSNephro is the first national research project on transitional care in the paediatric KTx population. Having all German paediatric transplant units involved in a single study offers the unique chance to gain insights into the existing structures and practice of transition across the country. This study uses the advantage of interview data, questionnaire and retrospective patient data complementing one another-and sometimes put one another into perspective.
A specific feature in Germany is that 16/21 departments belong to the non-profit organisation $\mathrm{KfH}$ and provide renal replacement therapy for children in association with university hospitals and large community hospitals. Although all participating institutions offer highly specialised care, the transition situation is remarkably heterogeneous and differs significantly between centres-even within the same organisation.

This study highlights that professionals working within paediatric nephrology in Germany are well aware of the importance of successful transition. Key elements of transitional care are well understood and mutually agreed on. Nonetheless, implementation within daily routine seems challenging, and the absence of written, structured procedures may hamper successful transition. Rigid regulations on transfer age as given by the relevant health authorities add on to this challenge.

In 2011, the International Society of Nephrology (ISN) and the IPNA published a consensus statement on transition. ${ }^{15}$ Nineteen components were considered essential; among others, these include the following: introduction of the concept of transition at age 12-14, avoidance of abrupt transfer, individualised transition, absence of crisis at transfer, completed school education, key worker coordinating transition ('champion'), generic transition plan and group sessions with other young people. The guidance provided is not specific to transplant recipients but is relevant for adolescents with all renal diseases. Other publications deal specifically with transition of young transplant recipients. ${ }^{16-18}$

Our interview data show that, as compared with these recommendations, the concept of transition is introduced quite late in the majority of German centres. While trying to achieve a highly individualised transition for every patient, this is bedevilled by a rigid age criterion which does not account for patients' needs and readiness. In some units, dedicated key workers coordinating transition ('champions') could be found, but this was not broadly established. After all, $86 \%$ of centres offered general in-house training courses, and the majority recommended participating in 'Endlich Erwachsen' (finally grown up) as a transition-specific programme. ${ }^{14}$ However, most healthcare professionals complained in the interviews that "those adolescents, who need this most, usually do not participate". Surprisingly, most German centres do not use a written transition plan to frame their action but rely on their experience and instincts.

Existing instruments to facilitate transition, such as a computer-based patient training and information system for adolescents with KTx, ${ }^{19}$ are not used in daily practice. Also, combined adolescent and young adult clinics to provide specialised care in times of transition ${ }^{20}$ are not established in Germany. An integrated multidisciplinary approach combined with peer support can optimise the management of young adults with KTx. ${ }^{21}$ Yet, the present administration and payment system does not account for such options. 
Existing regulations on transfer age as defined by the relevant administrative authorities are greatly disputed among professionals. All participants agreed that age 18 years as compulsory transfer age is arbitrary and does not account for the patients' medical needs, social framework or individual development including mental and biological maturity. Instead, such rules distract from focusing on a content-driven process, which is to prepare the young patients and help them achieve competence in self-care. ${ }^{15}$ The best available evidence suggests that patients with KTx transferred to adult care when older than 21 years have outcomes superior to those transferred at a younger age. ${ }^{22}$ Remarkably, adolescence is not the time with the highest rate of KTx failure-it is the time shortly after (age 19-23 years). ${ }^{5}$ Any regulation on transferring at age 18 years thus is neglecting the high-risk time of emerging adulthood.

It can be agreed on that there is no just 'one right time' for transition; rather, a flexible, individualised approach is needed to account for the patients' readiness. Social transitions such as leaving school and starting employment may be valuable surrogate parameters. Nevertheless, simply adjusting the regulations to allow for a higher transfer age is not effective either; rather, a thorough preparation of the adolescents is essential. The key question ought not to be 'how old is the patient?' but 'how well-prepared and equipped is the patient to move on into adult care?'

Assessing transition readiness is widely seen as an important component in optimising transition outcomes by identifying transition barriers, planning treatment individually and monitoring progress over time. ${ }^{23}{ }^{24}$ The ISN/IPNA consensus statement on transition ${ }^{15}$ provides the 'TR $\mathrm{T}_{\mathrm{X}} \mathrm{ANSITION}$ Scale' as an easily applicable tool to asses and monitor progress in achieving the goals of transition. The ability of the adolescent or young adult to provide own self-management and autonomy from parental care is assessed based on 10 components. Further tools to assess transition readiness have been developed and tested. ${ }^{23} 25-27$

Despite its importance and the availability of numerous tools and checklists, transition readiness is not routinely assessed in Germany. This is a problem, which is neither country specific ${ }^{28}$ nor disease specific. For example, a review of a large number of cystic fibrosis transition programmes (TPs) in the USA found the use of a list of desirable patient skills in less than $10 \%{ }^{29}$

The significant effectiveness of a multilevel TP on reduction of post-transfer episodes with acute rejection and transplant loss was recently demonstrated for German-speaking Switzerland. ${ }^{8}$ In addition, it is the first study showing the effect of a TP on estimated glomerular filtration rate 3 years after transfer. The Zurich TP starts at age 14 and includes a special adherence clinic, inclusion of the patient's school class, workshops for patients, parents and teachers, and a holiday camp. In contrast, a recent survey in paediatric nephrology centres of Japan revealed that only $4 \%$ had a TP at all and that $52 \%$ of patients were still followed up by paediatric renal services.

We conclude that, in German paediatric kidney transplant centres, awareness of transition issues exists, but its clinical implementation needs to be optimised. Structured TPs are rarely applied, and many readily available tools are not widely used.

The prospective, randomised interventional part of the TRANSNephro trial that is actually recruiting patients in all German centres for paediatric kidney transplantation is a unique chance to implement an improved transition routine. Obviously, the results of this trial have to be awaited before drawing final conclusions.

As a qualitative research project, the findings presented in this paper are based on the individual experiences of participating professionals and obviously influenced by personal opinions. As such, it serves as a base to explore the experiential aspects of transition and help generating more specific questions for further research.

To strengthen the credibility of this study, we interviewed several individuals with different professional backgrounds from the same department allowing us to gain multiple perspectives on the same setting.

Still, our report is limited to the perspective of medical doctors, nurses and psychosocial staff working in paediatric nephrology. We have not yet included the insights of adult nephrologists and the patients themselves. Additional research including those groups is needed to enhance our understanding of the present situation.

Given that we analysed a specific setting, that is, transition of young people with kidney transplantation in Germany, our findings are limited to this scene, and we cannot extrapolate our results to other geographical regions, healthcare settings or patient groups.

Having realised the gap between awareness of transition-related issues on the one hand and problems implementing structured care on the other hand, we need to identify barriers which keep professionals from establishing structured programmes and applying already available transition aids. The insight of this research will thus be used to design more detailed projects aiming to understand the multiple challenges of transition and ultimately help improving care.

\section{Author affiliations}

${ }^{1}$ Department of Paediatric Kidney, Liver and Metabolic Diseases, Hannover Medical School, Hannover, Germany

${ }^{2}$ Department of Epidemiology, Social Medicine and Health System Research, Hannover Medical School, Hannover, Germany

${ }^{3}$ Division of Paediatric Nephrology, Centre for Child and Adolescent Medicine, Heidelberg University Hospital, Heidelberg, Germany

${ }^{4}$ German Society of Transition Medicine, Hannover, Germany

${ }^{5}$ Berliner TransitionsProgramm (BTP), DRK-Kliniken (German Red Cross Hospitals) Berlin Westend, Berlin, Germany

${ }^{6}$ Department of Paediatric Nephrology, Charité, Berlin, Germany

${ }^{7}$ University Hospital of Bonn, Bonn, Germany

${ }^{8}$ Department of Paediatrics II, Essen University Hospital, Essen, Germany

${ }^{9}$ Children's Hospital, University of Erlangen, Erlangen, Germany

${ }^{10} \mathrm{KfH}$ Centre of Paediatric Nephrology, Clementine Children's Hospital, Frankfurt, Germany 
${ }^{11}$ Department of General Paediatrics, Adolescent Medicine and Neonatology, Freiburg University Hospital, Freiburg, Germany

${ }^{12}$ University Children's Hospital Eppendorf, Hamburg, Germany

${ }^{13}$ Division of Paediatric Nephrology, Centre for Child and Adolescent Medicine, Heidelberg University Hospital, Heidelberg, Germany

${ }^{14}$ University Children's Hospital, Jena, Germany

${ }^{15}$ Department of Paediatric Nephrology, University Hospital of Cologne, Cologne, Germany

${ }^{16}$ University Children's Hospital, Leipzig, Germany

${ }^{17} \mathrm{KfH}$ Centres of Paediatric Nephrology, St. Georg Hospital, Leipzig, Germany

${ }^{18} \mathrm{KfH}$ Centres of Paediatric Nephrology, University Hospital of Marburg, Marburg,

Germany

${ }^{19} \mathrm{KfH}$ Centre of Paediatric Nephrology, Children's Hospital Memmingen,

Memmingen, Germany

${ }^{20}$ University Children's Hospital Münster, Münster, Germany

${ }^{21} \mathrm{KfH}$ Centre of Paediatric Nephrology, University Children's Hospital, München,

Germany

${ }^{22}$ Dr.von Haunersches Kinderspital, Ludwigs Maximilian University, Munich,

Germany

${ }^{23}$ Children's Hospital, Olgahospital Klinikum Stuttgart, Stuttgart, Germany

${ }^{24}$ University Children's Hospital Tübingen, Tübingen, Germany

${ }^{25}$ University Children's Hospital, Rostock, Germany

${ }^{26} \mathrm{KfH}$ Centre of Nephrology, Hospitals of the Hannover Region, Hannover, Germany

${ }^{27}$ University Children's Hospital, Vienna, Austria

Correction notice This paper has been amended since it was published Online First. Owing to a scripting error, some of the publisher names in the references were replaced with 'BMJ Publishing Group'. This only affected the full text version, not the PDF. We have since corrected these errors and the correct publishers have been inserted into the references.

Acknowledgements We cordially thank the KfH Trust for Preventive Medicine for their generous funding which enabled this project. The present research would not have been possible without the kind help of all those doctors, nurses and psychosocial workers in the participating hospitals who provided access to data and enriched the manuscript with thoughtful input. We are thankful for all your support. Special gratitude goes to our study nurses Anna Tramm and Nicole Meyer for their ongoing commitment.

Contributors LP conceived and designed the study, drafted the study protocol, performed site visits and wrote the first draft of the paper. Dr MK designed the study, drafted the study protocol, performed site visits, evaluated and analysed the data, and wrote the first draft of the manuscript. J P designed the study, drafted the study protocol, performed the site visits, performed the interviews, transcribed, evaluated and analysed the data, and wrote the first draft of the paper. MLD designed the study. All authors contributed data to the research, revised the manuscript, approved the final manuscript as submitted and agreed to be accountable for all aspects of the work.

Competing interests None declared.

Ethics approval Hannover Medical School Ethics Committee and local committees of participating hospitals.

Provenance and peer review Not commissioned; externally peer reviewed.

Data sharing statement Interview guidelines can be obtained on request from the corresponding author. Transcripts cannot be forwarded for reasons of confidentiality.

Open Access This is an Open Access article distributed in accordance with the Creative Commons Attribution Non Commercial (CC BY-NC 4.0) license, which permits others to distribute, remix, adapt, build upon this work non-commercially, and license their derivative works on different terms, provided the original work is properly cited and the use is non-commercial. See: http://creativecommons.org/ licenses/by-nc/4.0/

(c) Article author(s) (or their employer(s) unless otherwise stated in the text of the article) 2017. All rights reserved. No commercial use is permitted unless otherwise expressly granted.

\section{REFERENCES}

1. White $\mathrm{PH}$, Ardoin S. Transitioning wisely: improving the connection from pediatric to adult health care. Arthritis Rheumatol 2016;68:789-94.
2. In: Bonnie RJ, Stroud $\mathrm{C}$, Breiner $\mathrm{H}$, eds. Investing in the health and well-being of young adults. authors: committee on improving the Health, Safety, and Well-Being of Young adults; Board on Children, Youth, and families; Institute of Medicine; National Research Council. Washington (DC): National Academies Press (US), 2015.

3. Kreuzer M, Prüfe J, Oldhafer M, et al. Transitional care and adherence of adolescents and young adults after kidney transplantation in Germany and Austria: a binational observatory census within the TRANSNephro trial. Medicine 2015;94:e2196.

4. Nehring WM, Betz CL, Lobo ML. Uncharted territory: systematic review of providers' roles, understanding, and views pertaining to health care transition. J Pediatr Nurs 2015;30:732-47.

5. Foster BJ. Heightened graft failure risk during emerging adulthood and transition to adult care. Pediatr Nephrol 2015;30:567-76.

6. Watson AR. Non-compliance and transfer from paediatric to adult transplant unit. Pediatr Nephrol 2000;14:0469-72.

7. Prestidge C, Romann A, Djurdjev O, et al. Utility and cost of a renal transplant transition clinic. Pediatr Nephrol 2012;27:295-302.

8. Weitz M, Heeringa S, Neuhaus TJ, et al. Standardized multilevel transition program: does it affect renal transplant outcome? Pediatr Transplant 2015;19:691-7.

9. Kreuzer M, Prüfe J, Bethe D, et al. The TRANSNephro-study examining a new transition model for post-kidney transplant adolescents and an analysis of the present health care: study protocol for a randomized controlled trial. Trials 2014;15:505.

10. Bloom SR, Kuhlthau K, Van Cleave J, et al. Health care transition for youth with special health care needs. $J$ Adolesc Health 2012;51:213-9.

11. Crowley R, Wolfe I, Lock K, et al. Improving the transition between paediatric and adult healthcare: a systematic review. Arch Dis Child 2011;96:548-53.

12. Grant $\mathrm{C}, \mathrm{Pan}$ J. A comparison of five transition programmes for youth with chronic illness in Canada. Child Care Health Dev 2011;37:815-20.

13. McDonagh JE, Kelly DA. The challenges and opportunities for transitional care research. Pediatr Transplant 2010;14:688-700.

14. John U, Offner G, Breuch K, et al. Konzept zur Verbesserung der Adhärenz bei jugendlichen erwachsenen nach nierentransplantation. Urologe 2009;48:1468-72.

15. Watson AR, Harden PN, Ferris ME, et al. Transition from pediatric to adult renal services: a consensus statement by the International Society of Nephrology (ISN) and the International Pediatric Nephrology Association (IPNA). Kidney Int 2011;80:704-7.

16. Bell LE, Sawyer SM. Transition of care to adult services for pediatric solid-organ transplant recipients. Pediatr Clin North Am 2010;57:593-610

17. LaRosa C, Glah C, Baluarte HJ, et al. Solid-organ transplantation in childhood: transitioning to adult health care. Pediatrics 2011;127:742-53.

18. Annunziato RA, Freiberger $\mathrm{D}$, Martin $\mathrm{K}$, et al. An empirically based practice perspective on the transition to adulthood for solid organ transplant recipients. Pediatr Transplant 2014;18:794-802.

19. Freier C, Oldhafer M, Offner G, et al. Impact of computer-based patient education on illness-specific knowledge and renal function in adolescents after renal transplantation. Pediatr Transplant 2010;14:596-602.

20. Harden PN, Walsh G, Bandler N, et al. Bridging the gap: an integrated paediatric to adult clinical service for young adults with kidney failure. BMJ 2012;344:e3718.

21. Harden PN, Sherston SN. Optimal management of young adult transplant recipients: the role of integrated multidisciplinary care and peer support. Ann Saudi Med 2013:33:489-91.

22. Foster BJ, Platt RW, Dahhou M, et al. The impact of age at transfer from pediatric to adult-oriented care on renal allograft survival. Pediatr Transplant 2011;15:750-9.

23. Marchak JG, Reed-Knight B, Amaral S, et al. Providers' assessment of transition readiness among adolescent and young adult kidney transplant recipients. Pediatr Transplant 2015;19:849-57.

24. Pai AL, Rausch J, Tackett A, et al. System for integrated adherence monitoring: real-time non-adherence risk assessment in pediatric kidney transplantation. Pediatr Transplant 2012;16:329-34.

25. Cantú-Quintanilla G, Ferris M, Otero A, et al. Validation of the UNC TRXANSITION Scale ${ }^{\mathrm{TM}}$ Version 3 among Mexican adolescents with chronic kidney disease. $J$ of Ped Nursing 2015;30:e71-e8.

26. Ferris $M$, Cohen $S$, Haberman $C$, et al. Self-management and transition readiness assessment: development, reliability, and factor structure of the STARx Questionnaire. J Pediatr Nurs 2015;30:691-9.

27. Cohen SE, Hooper SR, Javalkar K, et al. Self-management and transition readiness assessment: concurrent, predictive and discriminant validation of the STARx Questionnaire. J Pediatr Nurs 2015;30:668-76. 
28. Zhang LF, Ho JS, Kennedy SE. A systematic review of the psychometric properties of transition readiness assessment tools in adolescents with chronic disease. BMC Pediatr 2014;14:4.
29. McLaughlin SE, Diener-West M, Indurkhya A, et al. Improving transition from pediatric to adult cystic fibrosis care: lessons from a national survey of current practices. Pediatrics 2008;121:e116 $0-\mathrm{e} 1166$. 